\title{
Cyst infection in hospital-admitted autosomal dominant polycystic kidney disease patients is predominantly multifocal and associated with kidney and liver volume
}

\author{
B.E.P. Balbo ${ }^{1}$, M.T. Sapienza ${ }^{2}$, C.R. Ono ${ }^{2}$, S.K. Jayanthi ${ }^{3}$, J.B. Dettoni ${ }^{4}$, I. Castro ${ }^{1}$ and L.F. Onuchic ${ }^{1}$ \\ ${ }^{1}$ Divisão de Nefrologia, Faculdade de Medicina, Universidade de São Paulo, São Paulo, SP, Brasil \\ ${ }^{2}$ Divisão de Medicina Nuclear, Faculdade de Medicina, Universidade de São Paulo, São Paulo, SP, Brasil \\ ${ }^{3}$ Divisão de Radiologia, Faculdade de Medicina, Universidade de São Paulo, São Paulo, SP, Brasil \\ ${ }^{4}$ Divisão de Patologia, Faculdade de Medicina, Universidade de São Paulo, São Paulo, SP, Brasil
}

\begin{abstract}
Positron-emission tomography/computed tomography (PET/CT) has improved cyst infection (Cl) management in autosomal dominant polycystic kidney disease (ADPKD). The determinants of kidney and/or liver involvement, however, remain uncertain. In this study, we evaluated clinical and imaging factors associated with $\mathrm{Cl}$ in kidney $(\mathrm{KCl})$ and liver (LCI) in ADPKD. A retrospective cohort study was performed in hospital-admitted ADPKD patients with suspected Cl. Clinical, imaging and surgical data were analyzed. Features of infected cysts were evaluated by PET/CT. Total kidney (TKV) and liver (TLV) volumes were measured by CT-derived multiplanar reconstruction. $\mathrm{Cl}$ was detected in 18 patients who experienced 24 episodes during an interval of 30 months ( $\mathrm{LCl}$ in 12, $\mathrm{KCl}$ in 10 and concomitant infection in 2). Sensitivities of CT, magnetic resonance imaging and PET/CT were 25.0, 71.4, and 95.0\%. Dysuria $(P<0.05)$, positive urine culture $(P<0.01)$, and previous hematuria $(P<0.05)$ were associated with $\mathrm{KCl}$. Weight loss $(\mathrm{P}<0.01)$ and increased C-reactive protein levels $(\mathrm{P}<0.05)$ were associated with $\mathrm{LCl}$. $\mathrm{PET} / \mathrm{CT}$ revealed that three or more infected cysts were present in $70 \%$ of the episodes. TKV was higher in kidney-affected than in $\mathrm{LCl}$ patients (AUC $=0.91, \mathrm{P}<0.05)$, with a cut-off of $2502 \mathrm{~mL}(72.7 \%$ sensitivity, 100.0\% specificity). TLV was higher in liver-affected than in $\mathrm{KCl}$ patients $(\mathrm{AUC}=0.89, \mathrm{P}<0.01$ ) with a cut-off of $2815 \mathrm{~mL}(80.0 \%$ sensitivity, $87.5 \%$ specificity). A greater need for invasive procedures was observed in $\mathrm{LCl}(\mathrm{P}<0.01)$, and the overall mortality was $20.8 \%$. This study supports PET/CT as the most sensitive imaging method for diagnosis of cyst infection, confirms the multifocal nature of most hospitaladmitted episodes, and reveals an association of kidney and liver volumes with this complication.
\end{abstract}

Key words: Autosomal dominant polycystic kidney disease; Cyst infection; Positron-emission tomography/computed tomography; Kidney volume; Liver volume; Mortality

\section{Introduction}

Autosomal dominant polycystic kidney disease (ADPKD) is the most common monogenic kidney disorder, with an estimated prevalence of 1:500-1000 (1). The disease is characterized by bilateral and progressively enlarging kidney cysts, often leading to end-stage renal disease. In addition to kidney manifestations, a variety of extrarenal abnormalities can also occur, comprising cystic and non-cystic phenotypes (2).

Kidney infection in ADPKD includes acute pyelonephritis and/or cyst infection (Cl) (3). Although $\mathrm{Cl}$ tends to be associated with a defined area of tenderness and to be more insidious than parenchymal infection, both are typically manifested by fever and abdominal pain. This scenario often makes the distinction between these two clinical entities challenging (4). Liver $\mathrm{Cl}$ is also a common event in ADPKD, representing a potentially serious complication, and it can also occur in autosomal dominant polycystic liver disease (ADPLD) (5).

Conventional imaging methods have been shown to be of limited use in the diagnosis of renal or hepatic $\mathrm{Cl}$ (4). 
In recent years, however, the management of $\mathrm{Cl}$ in ADPKD has been redefined by the use of increasingly sophisticated nuclear medicine diagnostic techniques and the increased safety and effectiveness of image-guided, minimally invasive procedures that are sometimes required to drain infected foci, in addition to antibiotic therapy (ATB). The role of positron-emission tomography/computed tomography (PET/CT) has been analyzed in two retrospective series that support it as the gold standard imaging strategy for $\mathrm{Cl}$ diagnosis $(4,6)$. However, key questions and points remain unanswered and/or unaddressed (7), including the establishment of factors distinctly associated with kidney and liver $\mathrm{Cl}$, how often it affects multiple cysts and/or organs, to what extent this imaging strategy can be used in $\mathrm{Cl}$ management, and a proposal of how and when invasive procedures should be performed. To address these issues, we carried out a retrospective cohort study in hospitaladmitted ADPKD patients with clinically suspected $\mathrm{Cl}$.

\section{Material and Methods}

Study design, population and diagnostic criteria

This retrospective cohort study was conducted at Hospital das Clínicas, Universidade de São Paulo, São Paulo, SP, Brazil. The medical files of all ADPKD patients with suspected $\mathrm{Cl}$ who were admitted between May 2010 and November 2012 were reviewed, and relevant clinical, imaging, and invasive/surgical data were collected and categorized. ADPKD was diagnosed based on the criteria proposed by Pei et al. (8). Owing to the similar clinical presentation of $\mathrm{Cl}$ episodes, ADPLD patients with suspected liver $\mathrm{Cl}$ were also included in the medical file review. The diagnosis of ADPLD followed the criteria developed by Qian et al. (9).

The diagnosis of $\mathrm{Cl}$ was based on the criteria proposed by Sallée et al. (4) and was considered definite when cyst aspiration revealed microorganisms and/or neutrophil debris. We have extended these criteria by including tissue pathology analysis, from biopsy or autopsy, as an additional diagnostic tool. $\mathrm{Cl}$ was considered likely in the presence of fever (temperature $>38.5^{\circ} \mathrm{C}$ for $>3$ days), abdominal pain (particularly a palpable area of kidney or liver tenderness), increased C-reactive protein (CRP, >50 mg/L), absence of intracystic bleeding on CT, and exclusion of other inflammatory abdominal conditions. The patients were divided into two groups, definite and likely $\mathrm{Cl}$, and categorized according to the affected organ(s): kidney cyst(s) infection $(\mathrm{KCl})$, liver cyst(s) infection ( $\mathrm{LCl})$, and concomitant kidney and liver cyst(s) infection (KLCl; Figure 1).

$\mathrm{Cl}$ was considered severe in the presence of sepsis at admission, liver $\mathrm{Cl}$, or lack of improvement after 1 week of ATB therapy. The need for intervention was individualized. Image-guided drainage was performed in severe $\mathrm{Cl}$ cases as soon as possible, if feasible, regardless of cyst size. Open surgery was considered in cases with no clinical response to less invasive approaches. $\mathrm{Cl}$ resolution was

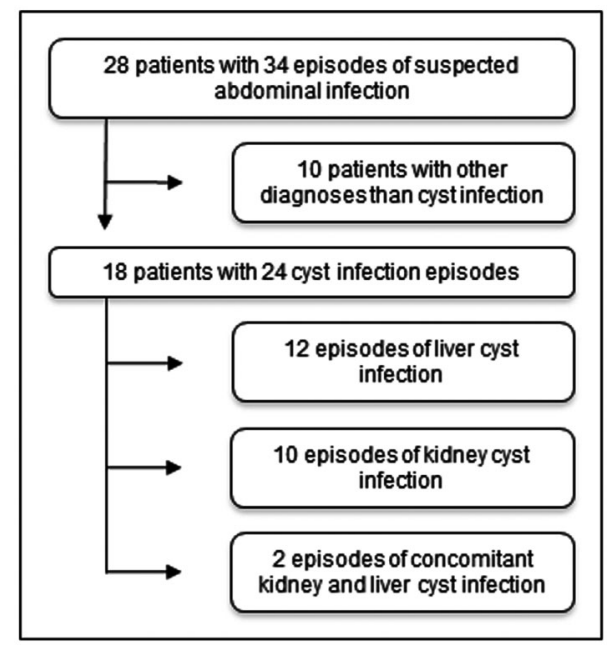

Figure 1. Retrospective cohort of hospital-admitted autosomal dominant polycystic kidney disease patients.

defined by disappearance of fever, CRP $<5 \mathrm{mg} / \mathrm{L}$ and at least two negative blood and/or urine cultures.

\section{Radiological analyses}

Kidney or liver ultrasound was considered positive for $\mathrm{Cl}$ based on detection of debris, thick wall and/or distal acoustic enhancement. CT scan was called positive for $\mathrm{Cl}$ when enhanced wall thickening and/or perilesional inflammation were observed. Recent intracystic bleeding was excluded by CT. The same criteria for detecting $\mathrm{Cl}$ were applied for magnetic resonance imaging (MRI), with the addition of marked reduction in diffusion on diffusionweighted MRI (10). When possible, contrast media were used for CT or MRI. The presence of air within cysts was indicative of emphysematous $\mathrm{Cl}$.

\section{Nuclear medicine analysis}

Suspected cases of $\mathrm{Cl}$ were further characterized by PET/CT, especially when ultrasound, CT and/or MRI were negative or inconclusive. A dedicated full-ring lutetium orthosilicate crystal-based scanner (Biograph 2, Siemens Medical Solutions, Germany, or Discovery 690, GE Healthcare, USA) was used. Studies were carried out 60-90 min after intravenous injection of $370 \mathrm{MBq}$ of ${ }^{18}$ fluorodeoxyglucose $\left({ }^{18} \mathrm{FDG}\right)$. Delayed images, when necessary, were obtained approximately $3 \mathrm{~h}$ after furosemide administration, except in anuric patients. Two independent, experienced nuclear medicine physicians (NMPs) reviewed the images. When consensus was not achieved, a third NMP opinion was obtained.

Attenuation-corrected PET/CT images were analyzed in transverse, coronal and sagittal planes and built into a three-dimensional maximum-intensity projection. Two patterns were considered positive for $\mathrm{Cl}$. The first was increased ${ }^{18} \mathrm{FDG}$ activity lining the cyst, in contrast to 
physiologic accumulation in parenchyma, and the second was diffuse signal accumulation within the cyst after exclusion of cyst hemorrhage by CT. Distinction between infection and pelvicalyceal activity, resultant from physiologic excretion of ${ }^{18} \mathrm{FDG}$, was sought by analyzing the decrease in activity following furosemide in delayed images.

Imaging features of each infectious episode, such as number of infected cyst(s) and location, were determined by PET/CT. Incidental findings, such as neoplasia or infection in other organs, were also analyzed. The ${ }^{18}$ FDG level was measured by the maximum-standardized uptake value $\left(S U V_{\max }\right)$, determined by drawing regions of interest in the attenuation-corrected ${ }^{18}$ FDG-PET images around the suspected lesion (6). A third NMP opinion was requested for SUV $_{\text {max }}$ differences $>10 \%$. Adequate glycemic control was checked before each examination.

\section{Organ and cyst measurements}

Total kidney (TKV) and liver (TLV) volumes were measured using CT-derived multiplanar reconstruction, with DICOM images processed by the OsiriX software (11). Evaluation of infected cyst size was also performed using CT, by measuring the largest internal diameter of each infected cyst. In cases of multiple $\mathrm{Cl}$, the three largest infected cysts were included in the analysis.

\section{Statistical analysis}

Bivariate analysis was performed by the Pearson chisquare test for categorical data and reported as frequency and percentage. The Kolmogorov-Smirnov test was used to determine normality for continuous data. Differences between two samples were compared using the unpaired Student $t$-test, with data reported as means $\pm S D$. Nonparametric data were compared by the Mann-Whitney test, with results reported as medians and percentiles. Variable discrimination was calculated using receiver operating characteristic ( $\mathrm{ROC}$ ) curves, assessing the area under the curve and asymptotic significance. Significance level was set at $5 \%(P=0.05)$. The SPSS v.19.0 Statistical Package (IBM Corp., USA) was used for analyses.

\section{Results}

\section{Clinical evaluation and characterization of $\mathrm{Cl}$ events}

Thirty-four episodes of suspected abdominal infection were identified in 27 ADPKD and 1 ADPLD subjects. Ten ADPKD patients had a diagnosis other than $\mathrm{Cl}$. In this group, $\mathrm{Cl}$ was excluded based not only on the absence of PET/CT imaging criteria but also by the establishment of alternative diagnoses supported by imaging and/or clinical findings. In those 10 cases, four patients presented kidney cyst hemorrhage, one had liver cyst rupture, and two had fever of unknown origin despite extensive workup. Each of the following diagnoses were detected in one patient: vertebral osteomyelitis detected by PET/CT; acute cholecystitis, not initially seen on CT scan but diagnosed with PET/CT; and retroperitoneal abscess detected months after a unilateral nephrectomy because of chronic kidney pain. Twelve of the 18 remaining patients completed treatment with cure, 5 died from $\mathrm{Cl}$, and $1 \mathrm{LCl}$ patient died intraoperatively during liver transplantation.

Reinfection occurred in 6 patients, a high recurrence rate. All patients had fever and abdominal pain at admission. An association between dysuria and $\mathrm{KCl}$ was observed $(P<0.01)$, while a history of recent weight loss was more frequently associated with $\mathrm{LCl}(\mathrm{P}<0.01$; Table 1). CRP levels were higher in $\mathrm{LCl}$ than in $\mathrm{KCl}$ episodes $(P<0.05$; Table 2). No significant differences in estimated glomerular filtration rate (GFR using MDRD equation) or previous history of renal replacement therapy were observed between groups (Table 2). Positive blood cultures were obtained in $\mathrm{KCl}$ and $\mathrm{LCl}$, but only $\mathrm{KCl}$ patients presented positive urine cultures (Table 2).

A high prevalence of recurrent gross hematuria and hypertension was observed in this patient population (Supplementary Table S1). The initial infectious foci,

Table 1. Clinical manifestations at hospital admission in patients with autosomal dominant polycystic kidney disease/autosomal dominant polycystic liver disease with kidney and/or liver cyst infections.

\begin{tabular}{lcccc}
\hline Signs/symptoms & $\mathrm{KCl}$ & $\mathrm{LCl}$ & $\mathrm{KLCl}$ & All episodes \\
\hline Abdominal pain & $10(100)$ & $12(100)$ & $2(100)$ & $24(100)$ \\
Fever & $10(100)$ & $12(100)$ & $2(100)$ & $24(100)$ \\
$\geq 10 \%$ weight loss & $1(10)$ & $6(50)^{\mathrm{a}}$ & $1(50)$ & $8(33.3)$ \\
Gross hematuria & $3(30)$ & $1(8.3)$ & 0 & $4(16.7)$ \\
Dysuria & $7(70)^{\mathrm{b}}$ & 0 & 0 & $7(29.2)$ \\
Diarrhea & $3(30)$ & $2(25)$ & $1(50)$ & $7(29.2)$ \\
Respiratory symptoms & $2(20)$ & $9(75)$ & $1(50)$ & $4(16.7)$ \\
Sepsis & $4(40)$ & & & $14(58.3)$
\end{tabular}

Data are reported as number with percent in parentheses. KCl: kidney cyst infection; LCl: liver cyst infection; KLCl: concomitant kidney and liver cyst infection. ${ }^{\mathrm{a}} \mathrm{P}<0.05$ vs $\mathrm{KCl}$ group; ${ }^{\mathrm{b}} \mathrm{P}<0.01$ vs $\mathrm{LCl}$ group (Pearson chi-square test). 
Table 2. Clinical features of cyst infection episodes in patients with autosomal dominant polycystic kidney disease/autosomal dominant polycystic liver disease.

\begin{tabular}{|c|c|c|c|c|}
\hline Characteristics & $\mathrm{KCl}$ & $\mathrm{LCl}$ & $\mathrm{KLCl}$ & All groups \\
\hline Patient and episode ${ }^{+}$ & $9(10)$ & $10(12)$ & $2(2)$ & $18(24)^{+}$ \\
\hline $\begin{array}{l}\text { Patient age [years; } \\
\text { median (p25-p75)] }\end{array}$ & $49.5(42.5-52.0)$ & $53.0(42.0-56.0)$ & 52 and 40 & $51.0(42.0-55.0)$ \\
\hline $\begin{array}{l}\mathrm{GFR}\left[\left(\mathrm{mL} \cdot \mathrm{min}^{-1} \cdot 1.73\left(\mathrm{~m}^{2}\right)^{-1}\right)\right. \\
\quad \operatorname{mean} \pm \mathrm{SD}]\end{array}$ & $27.4 \pm 26.9$ & $31.1 \pm 24.4$ & 25.0 for both & $29.0 \pm 23.9$ \\
\hline On dialysis [n (\%)] & $3(30.0)$ & $3(25.0)$ & 0 & $6(25.0)$ \\
\hline $\begin{array}{l}\text { Leukocyte count } \\
{\left[\mathrm{mm}^{3} ; \text { median (p25-p75)] }\right.}\end{array}$ & $12,975(7,800-27,830)$ & $8,820(7,525-26,715)$ & $\begin{array}{l}28,830 \text { and } \\
11,030\end{array}$ & $11,030(7,690-26,715)$ \\
\hline $\begin{array}{l}\text { CRP }[\mathrm{mg} / \mathrm{L} ; \text { median } \\
(\mathrm{p} 25-\mathrm{p} 75)]\end{array}$ & $222(132-250)$ & $306^{a}(248-332)$ & 266 and 53 & $256(182-313)$ \\
\hline Urine culture $(n)^{++}$ & E. coli (6), K. pneumonie (1) ${ }^{\mathrm{b}}$ & Negative & Negative & $7(30.4 \%)$ \\
\hline Blood culture (n) & E. coli (4), C. parapsilosis (1) & E. coli (2), E. cloacae (1) & E. coli (1) & $9(37.5 \%)$ \\
\hline $\begin{array}{l}\text { Cyst aspiration } \\
\text { culture }(n)\end{array}$ & S. corvalis (1), E. coli (1) & $\begin{array}{l}\text { O. antropi (1), S. aureus (1), } \\
\text { E. coli }(1), \text { R. planticola (1) }\end{array}$ & Negative & 6 in $15(40.0 \%)$ \\
\hline $\begin{array}{l}\text { Ciprofloxacin as first } \\
\text { ATB }[n(\%)]\end{array}$ & $6(60.0)$ & $7(58.3)$ & $2(100.0)$ & $15(62.5)$ \\
\hline Invasive procedure $(\mathrm{n})$ & IGPD (2), Nx (4) & $\begin{array}{l}\text { IGPD (9), liver Tx after } \\
\text { unsuccessful IGPD (1) }\end{array}$ & IGPD (1) & 17 episodes $(70.8 \%)$ \\
\hline $\begin{array}{l}\text { ATB duration } \\
\quad(\text { weeks; mean } \pm \text { SD })^{+++}\end{array}$ & $10.3 \pm 4.5$ & $10.8 \pm 3.5$ & 8 for both & $10.3 \pm 3.6$ \\
\hline $\begin{array}{l}\text { Deaths directly related to } \\
\text { cyst infection [n (\%)] }\end{array}$ & $2(20.0)$ & $3(25.0)$ & None & $5(20.8)$ \\
\hline
\end{tabular}

KCl: kidney cyst infection; LCl: liver cyst infection; KLCl: concomitant kidney and liver cyst infection; GFR: glomerular filtration rate; CRP: C-reactive protein; ATB: antibiotic; IGPD: image-guided percutaneous drainage; Nx: nephrectomy; Tx: transplantation; p25-p75: 25 to 75 percentile. ${ }^{+}$One patient with $\mathrm{KCl}$ also had an episode of $\mathrm{LCl}$; both $\mathrm{KLCl}$ patients also had an $\mathrm{LCl}$ episode (Figure 1). ${ }^{++}$Urine culture was available in 23 of 24 episodes (all but 1 anuric patient on dialysis). ${ }^{++}$Includes patients who achieved cure and excludes those for whom ATB was interrupted due to death. ${ }^{a} \mathrm{P}<0.05$ vs $\mathrm{KCl}$ group (Mann-Whitney test); ${ }^{\mathrm{b}} \mathrm{P}<0.01 \mathrm{vs} \mathrm{LCl}$ group (Pearson chisquare test).

before $\mathrm{Cl}$ diagnosis, were attributed to a myriad of locations (Supplementary Table S2); while urinary tract involvement occurred exclusively in $\mathrm{KCl}$, the source was not identified in $16.7 \%$ of the episodes, all of which were $\mathrm{LCI}$.

\section{Management and decision making in $\mathrm{Cl}$ events}

All patients with suspected $\mathrm{Cl}$ received ATB. Intravenous ciprofloxacin, alone or in combination, was the preferred option prior to obtaining culture results (Table 2). A decision for combination ATB was made in $75 \%$ of the episodes, particularly in severe presentations. All surviving patients received ATB for at least 4 weeks, and $66.7 \%$ required 8 or more weeks to reach cure criteria. Change to trimethoprim-sulfamethoxazole (5 cases) or chloramphenicol (1 case), supported by an antibiogram, was made to achieve cure. An apparent higher prevalence of sepsis was observed in $\mathrm{LCl}$ than in $\mathrm{KCl}$ events.

Escherichia coli was isolated in blood culture in $29.2 \%$ of all episodes and in urine culture in $25 \%$ (Table 2). Salmonella corvalis was detected by cyst drainage in one patient following a diarrheal episode, suggesting translocation; the identification of a quinolone-resistant microorganism prompted change to trimethoprim-sulfamethoxazole.
Image-guided percutaneous drainage (IGPD) was performed in 10 of $12 \mathrm{LCl}$ episodes (Table 2). Need for intervention was also observed in $\mathrm{KCl}$, including two IGPD and four nephrectomies. The retrospective analysis also detected one case of ADPLD that developed $\mathrm{LCl}$ with multiple infected cysts following liver fenestration. Cure came after effective IGPD, which revealed $S$. aureus and guided change to vancomycin. Moreover, in seven episodes, the diagnosis of $\mathrm{Cl}$ was established by tissue pathology of the removed organ or at autopsy (Figure $2 \mathrm{H}$ and I).

\section{Diagnostic impact of imaging methods}

All patients were evaluated by $\mathrm{CT}$, with an overall diagnostic sensitivity of $25 \%$ (Figure 3 ). Seven CTnegative cases were evaluated by MRI. The result was positive in 5 and negative in 2. While the positive MRI results were confirmed by $\mathrm{PET} / \mathrm{CT}$, this exam also revealed that the negative MRIs were false, resulting in an overall sensitivity of $71.4 \%$ (Figure $2 \mathrm{~A}-\mathrm{C}$ ). PET/CT was performed in $20 \mathrm{Cl}$ episodes, with an overall sensitivity of 95\% (Figure 3). Diagnostic discordance was limited to a single case that was considered positive by a third NMP and confirmed as $\mathrm{Cl}$ by pathology following nephrectomy. 

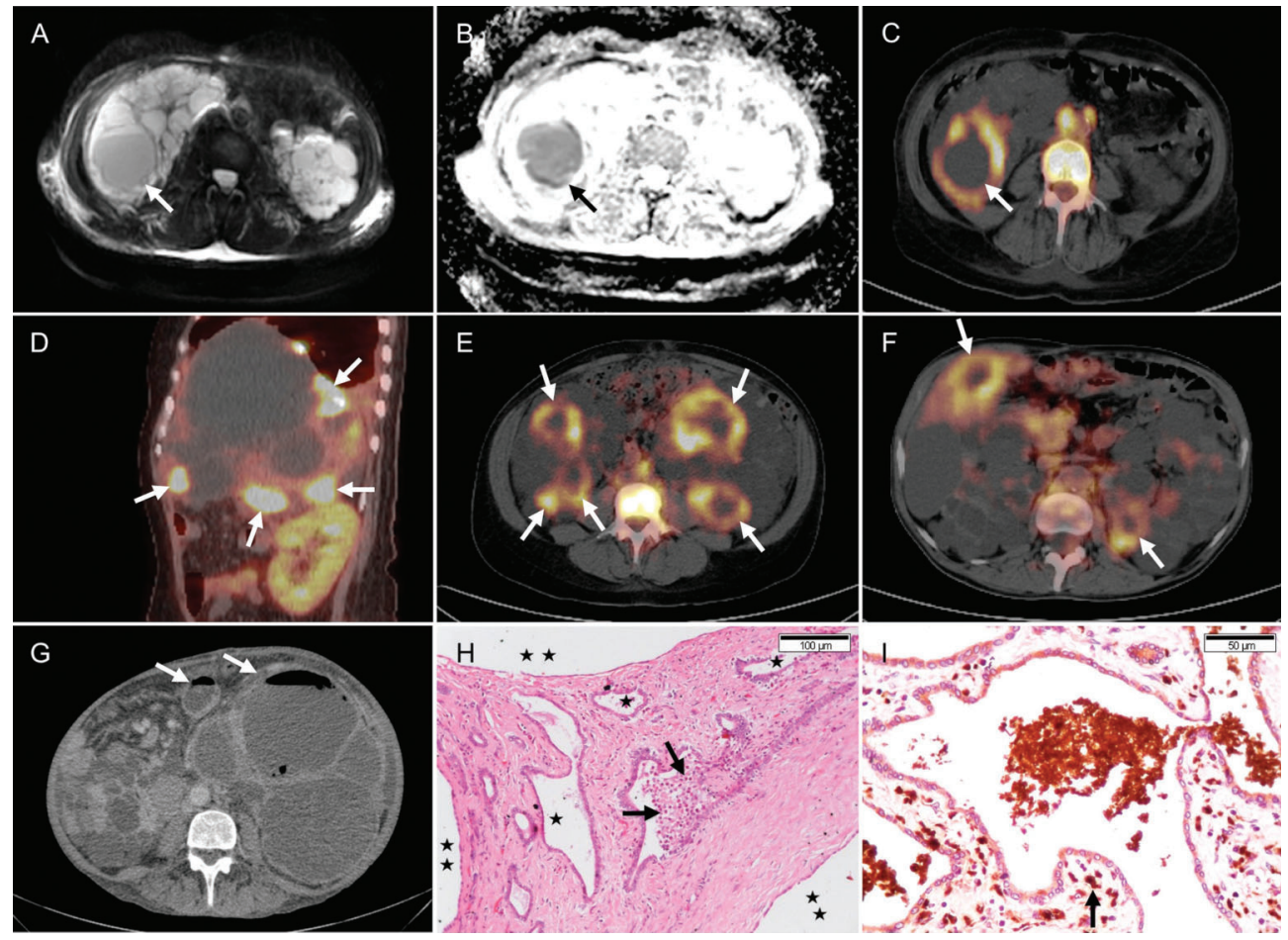

Figure 2. Cyst infection in autosomal dominant polycystic kidney disease/autosomal dominant polycystic liver disease (ADPLD) documented by several imaging methods $(A-G)$ and histology $(H-I)$. $A-C$, chronic kidney disease (CKD) stage 5 patient with kidney cyst infection $(\mathrm{KCl})$. $A$, T2-weighted magnetic resonance imaging (MRI). Infected cyst in right kidney, axial plane (arrow). B, Diffusionweighted MRI (DWMRI). Infected cyst in right kidney shows restriction to diffusion (arrow). $C$, positron-emission tomography/computed tomography (PET/CT). High correlation between T2-weighted MRI $(A)$, DWMRI $(B)$ and PET/CT in a CKD stage 5 patient with KCI (arrow). $D, P E T / C T$. Multiple infected liver cysts after cyst fenestration in an ADPLD patient (arrows). E, PET/CT. Bilateral renal infected cysts (arrows), coronal plane. Imaging analysis reveals increased cyst-lining ${ }^{18}$ FDG activity and signal accumulation within the cyst (inferior pole, right kidney). F, PET/CT. Concomitant kidney and liver cyst infection (arrows), axial plane. G, CT scan. Left kidney with emphysematous cyst infection (arrows), axial plane. $H$, Small liver cyst filled with neutrophils (arrows), small cysts containing no neutrophils (star), and large cysts lined by flat epithelium with no neutrophils (double stars; H\&E, original magnification, $200 \times$, bar: $100 \mu \mathrm{m}) . I$, Myeloperoxidase immunohistochemistry showing positive signal in neutrophils surrounding fibrous stroma (arrow) and within the cyst (original magnification, $400 \times$, bar: $50 \mu \mathrm{m}$ ).

Our calculations showed, for our sample size of 24 events, a $>95 \%$ power to detect $\mathrm{Cl}$ by comparing CT with $\mathrm{PET} / \mathrm{CT}$ and CT with $\mathrm{MRI}$, and a $>80 \%$ power by comparing MRI with PET/CT.

\section{Cyst infection and multifocality}

Cyst infection was predominantly multifocal (Figures 4 and $2 \mathrm{D}-\mathrm{F}$ ). A single $\mathrm{Cl}$ occurred in only $15 \%$ of the events, but three or more infected cysts were detected in $70 \%$ of all episodes analyzed by PET/CT. Interestingly, in $\mathrm{KCl}$ limited to one kidney, the dominant organ was affected in the majority of the cases (Table 3). However, bilateral involvement was not uncommon in the $\mathrm{KCl}$ group, being detected in $30 \%$ of the cases. Another remarkable observation was the fact that the majority of the infectious episodes affected nondominant cysts. The involvement of the dominant cyst, as shown by all image data combined, was in fact limited to $40 \% \mathrm{KCl}, 25 \% \mathrm{LCl}$, no $\mathrm{KLCl}$, and $29.2 \%$ overall.

\section{PET/CT features and incidental findings}

The PET/CT analyses detected two $\mathrm{Cl}$ imaging patterns. The first, characterized by increased ${ }^{18} \mathrm{FDG}$ activity lining the cyst, was more prevalent and occurred in $85 \%$ of $\mathrm{Cl}$ episodes (Figure 2C, E, and $\mathrm{F}$ ). The second was characterized by diffuse ${ }^{18} \mathrm{FDG}$ signal accumulation within the cyst and occurred in $60 \%$ of $\mathrm{Cl}$ episodes (Figure $2 \mathrm{D}$ and $\mathrm{E}$ ). The mean $\mathrm{SUV}_{\max }$ was $6.6 \pm 1.2$ in $\mathrm{KCl}$ episodes, $7.9 \pm 3.2$ in $\mathrm{LCl}$ episodes and $6.9 \pm 2.4$ in all events combined.

Concomitant episodes of pneumonia, aortitis and subphrenic abscess were also revealed by PET/CT. Incidental neoplastic findings included gastric and thyroid cancer. In all $\mathrm{Cl}$ events, PET/CT was performed at a median of 12 days ( 7.0 to 20.5 days) after initiating ATB. This method yielded a false-negative result in only one case, where LCl was confirmed at autopsy (Supplementary Table S3). MRI, in turn, had intermediate sensitivity for $\mathrm{Cl}$ detection (Figure 3). 


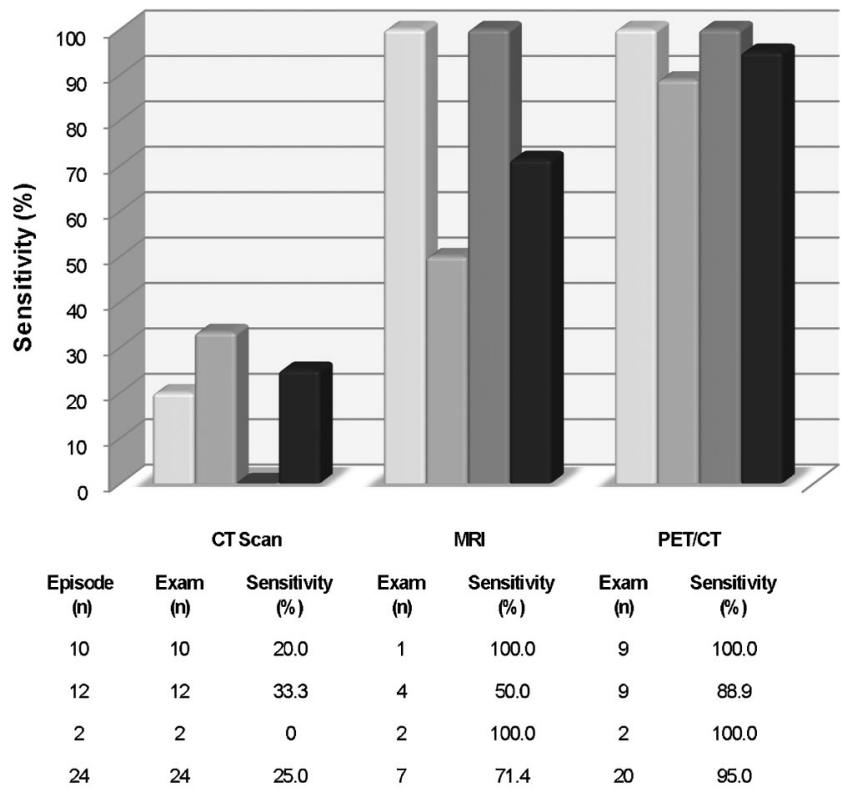

Figure 3. Sensitivity of different imaging methods to detect cyst infection in autosomal dominant polycystic kidney disease/autosomal dominant polycystic liver disease. CT: computed tomography; MRI: magnetic resonance imaging; PET: positron-emission tomography; $\mathrm{KCl}$ : kidney cyst infection; LCl: liver cyst infection; KLCl: concomitant kidney and liver cyst infection.

\begin{tabular}{lccccccc} 
Group & $\begin{array}{c}\text { Episode } \\
(\mathbf{n})\end{array}$ & $\begin{array}{c}\text { Exam } \\
(\mathbf{n})\end{array}$ & $\begin{array}{c}\text { Sensitivity } \\
(\%)\end{array}$ & $\begin{array}{c}\text { Exam } \\
(\mathbf{n})\end{array}$ & $\begin{array}{c}\text { Sensitivity } \\
(\%)\end{array}$ & $\begin{array}{c}\text { Exam } \\
(\mathbf{n})\end{array}$ & $\begin{array}{c}\text { Sensitivity } \\
(\%)\end{array}$ \\
\hline KCl & 10 & 10 & 20.0 & 1 & 100.0 & 9 & 100.0 \\
\hline LCl & 12 & 12 & 33.3 & 4 & 50.0 & 9 & 88.9 \\
\hline KLCl & 2 & 2 & 0 & 2 & 100.0 & 2 & 100.0 \\
All groups & 24 & 24 & 25.0 & 7 & 71.4 & 20 & 95.0
\end{tabular}

Cyst infection associates with kidney and liver volume

Patients with $\mathrm{KCl}$, isolated or combined with $\mathrm{LCl}$, had higher TKVs than ADPKD cases with isolated $\mathrm{LCl}$. The area under the ROC (AUC) was 0.91 [95\% confidence interval $(95 \% \mathrm{Cl})=0.76-1.06, \mathrm{P}=0.013$; Figure $5 \mathrm{~A}]$. The highest accuracy point detected was for TKV $=2502 \mathrm{~mL}$, with $72.7 \%$ sensitivity and $100.0 \%$ specificity (Figure $5 B$ ). $\mathrm{KCl}$ was also associated with HtTKV (height-adjusted TKV, a potential prognostic biomarker (12); $P=0.013$ ). In this case, the highest accuracy point detected was for

HtTKV $=1599 \mathrm{~mL} / \mathrm{m}$. Similarly, patients with $\mathrm{LCl}$, isolated or combined with $\mathrm{KCl}$, had higher TLVs compared with isolated $\mathrm{KCl}$ subjects $(\mathrm{AUC}=0.89,95 \% \mathrm{Cl}=0.72-1.05$, $\mathrm{P}=0.004$; Figure $5 \mathrm{C})$. The highest accuracy point detected was for TLV $=2815 \mathrm{~mL}$, with $80.0 \%$ sensitivity and $87.5 \%$ specificity (Figure $5 \mathrm{D}$ ).

\section{Most hospital-admitted episodes of $\mathrm{Cl}$ require invasive procedures}

Eleven $\mathrm{Cl}$ episodes were associated with $\geq 5-\mathrm{cm}$

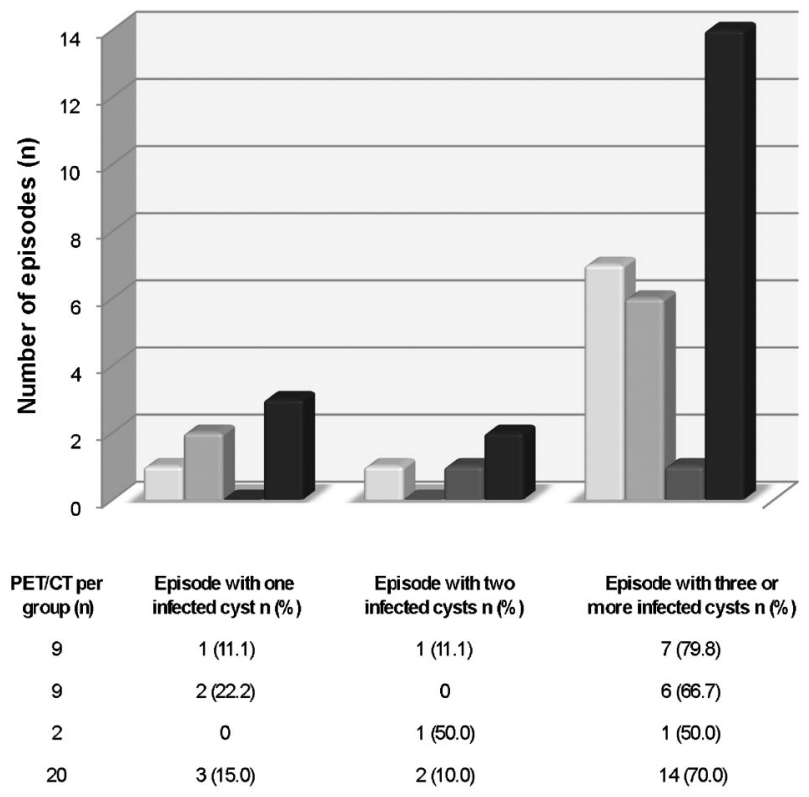

Figure 4. Number of infected cysts per episode evaluated by positron-emission tomography/ computed tomography (PET/CT). KCl: kidney cyst infection; LCl: liver cyst infection; KLCl: concomitant kidney and liver cyst infection. $\dagger$ : one ADPLD case. 
Table 3. Organ volume and anatomical analysis in autosomal dominant polycystic kidney disease (ADPKD)/autosomal dominant polycystic liver disease (ADPLD) patients with cyst infection.

\begin{tabular}{|c|c|c|c|}
\hline Group & $\mathrm{KCl}$ & $\mathrm{LCl}$ & Total \\
\hline TKV per patient $^{+}(\mathrm{mL})$ & $4210(1972-5803)^{a}$ & $1814(962-2773)$ & $2502(1579-4556)$ \\
\hline $\mathrm{TLV}_{\text {per patient }}{ }^{+}(\mathrm{mL})$ & $1842(1608-2472)$ & $4470(2824-9081)^{b}$ & $2816(1842-5832)$ \\
\hline Cyst diameter per episode ${ }^{+++}(\mathrm{cm})$ & $5.2(3.4-5.4)$ & $4.2(3.2-6.5)$ & $5.2(3.4-7.7)$ \\
\hline Infection in dominant cyst/episode & $4 / 10(40.0 \%)$ & $3 / 12(25.0 \%)$ & $7 / 24(29.2 \%)$ \\
\hline Cyst infection in dominant kidney/episode & $5 / 7(71.4 \%)$ & Not applicable & $7 / 9(77.8 \%)$ \\
\hline Concomitant cyst infection in both kidneys/episode & $3 / 10(30.0 \%)$ & Not applicable & $3 / 12(25 \%)$ \\
\hline
\end{tabular}

Data are reported as median with p25-p75 in parentheses or as number with percent in parentheses. TKV: total kidney volume; TLV: total liver volume; KCl: kidney cyst infection; LCl: liver cyst infection; p25-p75: 25 to 75 percentile. ${ }^{+}$An ADPKD patient with previous unilateral nephrectomy and 1 ADPLD subject were excluded from the TKV analysis. ${ }^{++}$The ADPLD patient was included in the TLV analysis. ${ }^{+++}$The largest internal diameter of each infected cyst was measured by computed tomography. In each episode, when multiple cysts were infected, the three largest cysts were measured. ${ }^{a} \mathrm{P}<0.05$ vs $\mathrm{LCl}$ group; ${ }^{\mathrm{b}} \mathrm{P}<0.01$ vs $\mathrm{KCl}$ group (Mann-Whitney test).

infected cysts $(6 \mathrm{KCl}, 4 \mathrm{LCl}$, and $1 \mathrm{KLCl})$. Seven of them were successfully treated by invasive procedures and 3 achieved cure with only ATB. One was a late referral, evolving to death before invasive intervention was performed. In contrast, $13 \mathrm{Cl}$ episodes were associated with
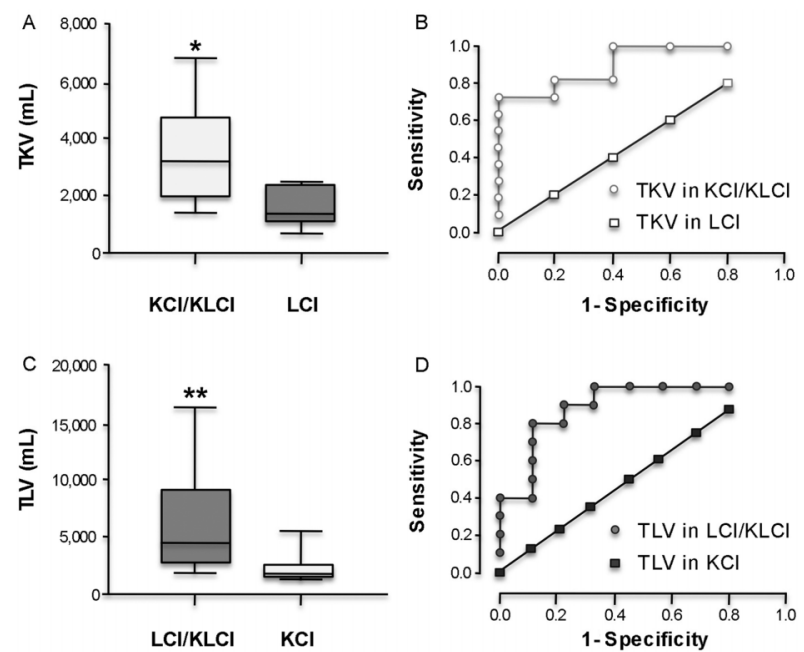

Figure 5. Association between organ volume and cyst infection. $A$, Patients admitted to the hospital with $\mathrm{KCl} / \mathrm{KLCl}$ presented higher TKV in comparison with those with isolated LCl [3491 mL (21365167; p25-p75) vs 1021 mL (745-2157; p25-p75)] ( ${ }^{*}<0.05$, MannWhitney test). $B$, Receiver operating characteristic curve (ROC) analysis for TKV. The highest accuracy point for $\mathrm{KCl}$ was TKV $=2502 \mathrm{~mL}$, with $72.7 \%$ sensitivity and $100.0 \%$ specificity (AUC $=0.91,95 \% \mathrm{Cl}=0.76-1.06, \mathrm{P}=0.013$ ). C, Patients with LCl/ $\mathrm{KLCl}$ displayed higher TLV compared with those with isolated $\mathrm{KCl}$ [4470 mL (2824-9081; p25-p75) vs 1842 mL (1570-2527; p25-p75)] ${ }^{* *} \mathrm{P}<0.01$, Mann-Whitney test). $D, \mathrm{ROC}$ analysis for TLV. The highest accuracy point for liver cyst infection was TLV $=2815 \mathrm{~mL}$, with $80.0 \%$ sensitivity and $87.5 \%$ specificity ( $A \cup C=0.89$, $95 \% \mathrm{Cl}=0.72-1.05, \mathrm{P}=0.004) . \mathrm{KCl}$ : kidney cyst infection; $\mathrm{LCl}$ : liver cyst infection; KLCl: concomitant kidney and liver cyst infection; TKV: total kidney volume; TLV: total liver volume. $<5$-cm infected cysts ( $4 \mathrm{KCl}, 8 \mathrm{LCl}$, and $1 \mathrm{KLCl}$ ). Ten were treated by invasive interventions, 7 of which were successful; 1 episode was cured with ATB only, and 2 patients died before the invasive procedure. One was a late referral and one was a postmortem diagnosis. In these cases, therefore, the vast majority of hospital-admitted patients required invasive intervention, including $<5-\mathrm{cm}$ infected cysts. The details of patients who died are shown in Supplementary Table S3.

\section{Carbohydrate antigen 19-9 (CA19-9) and liver $\mathrm{Cl}$}

CA19-9 was evaluated in $1 \mathrm{KLCl}, 4 \mathrm{KCl}$ and $11 \mathrm{LCl}$ episodes. Using the proposed cut-off of $106 \mathrm{U} / \mathrm{mL}$ (13), CA19-9 failed to support the diagnosis of $\mathrm{LCl}$ in 6 episodes (54.5\%).

\section{PET/CT for control of treatment efficacy}

A second PET/CT analysis was performed in four patients as a control evaluation. Notably, it changed decision-making in two of them. In a patient with previous $\mathrm{KLCl}$ and gastric cancer, the absence of $\mathrm{Cl}$ signs allowed for chemotherapy administration. In another case with multiple infected cysts and preclusion of IGPD due to technical difficulties, a positive PET/CT supported extending ATB therapy for seven additional weeks despite the relative decrease in $\mathrm{CRP}$ levels that occurred before the second imaging examination. In the other two cases, it confirmed $\mathrm{Cl}$ cure in patients with borderline clinical status but did not change overall decision-making.

\section{Discussion}

The diagnosis of $\mathrm{Cl}$ in ADPKD is often challenging $(4,6,7)$. In this scenario, attempts to define diagnostic criteria based on imaging techniques have been proposed (14-18). Conventional imaging, however, and even gallium or indium scanning, have low sensitivity $(3,19)$. Newer imaging strategies, together with the increasing safety and effectiveness of minimally invasive procedures, have 
redesigned the management of such a potentially devastating complication (20). Recent reports suggest that PET/ $\mathrm{CT}$ is the best tool for $\mathrm{Cl}$ detection $(4,6)$, a finding that we have confirmed in our series. Despite PET/CT availability in our center, this retrospective cohort reflected an effort toward accurate diagnosis balanced against cost-effectiveness. For that reason, CT was used as the first imaging approach in all suspected $\mathrm{Cl}$ episodes. Compared with MRI and PET/CT, CT offers the shortest waiting time, relatively low cost, and high accuracy to exclude nephrolithiasis and cyst hemorrhage. On the other hand, MRI with diffusionweight acquisition, emerges as a potentially reliable tool, apparently with higher sensitivity for $\mathrm{Cl}$ detection than $\mathrm{CT}$. This observation is, however, limited by the low number of patients with MRI results, and merits further study.

Like others, we have been confronted with the clinical dilemma imposed by difficulties to accurately determine $\mathrm{Cl}$. The confirmation of such a diagnosis was achieved following the criteria proposed by Sallée et al. (4), by searching for neutrophil debris and/or microorganisms, a procedure only feasible when drainage was performed. To improve sensitivity, we have broadened the definite diagnosis criteria to include $\mathrm{Cl}$ episodes documented by pathology evaluation, when available.

Our patients had multiple comorbid conditions, including risk factors for infection. In the current series, the numbers of $\mathrm{LCl}$ and $\mathrm{KCl}$ episodes were similar. Interestingly, in these two groups, patient age and need for renal replacement therapy did not differ.

Fever and abdominal pain were universal at admission. The search for attributable infectious foci was based on the assumption that a primary infection precedes $\mathrm{Cl}$ eclosion. Indeed, in the present study, the primary site was detected in $83.3 \%$ of all episodes. The observation that urinary tract manifestations were common in $\mathrm{KCl}$ and associated with a high prevalence of Gram-negative bacteria supports the concept that the ascending route was the predominant source of infection in this group. The hematogenous spread from endocarditis and a probable intestinal translocation of Salmonella corvalis, support alternative sources for kidney $\mathrm{Cl}$. Moreover, the high reinfection rate suggests a prominent role of anatomical abnormalities in kidney and liver for determining $\mathrm{Cl}$ (21).

We have performed quantitative evaluation of the number of infected cysts, as well as volumetric analyses of kidneys and livers, an original approach not performed in previously published series $(4,6)$. Our data showed that $\mathrm{Cl}$ was predominantly multifocal in both kidney and liver. These results were supported not only by PET/CT imaging but also by drainage and histopathologic data confirming multiple affected cysts. Hematogenous spread, low oxygen tension within the cyst wall, and anatomical abnormalities in ADPKD/ADPLD could contribute to the creation of niches for infection development. Our study indicates, therefore, that diagnosis of $\mathrm{Cl}$ in one kidney does not preclude its occurrence in the contralateral kidney or even in the liver.

Remarkably, the dominant cyst was infected in only $29.2 \%$ of $\mathrm{Cl}$ episodes, indicating that a search focused on it can often be misleading. In addition, the size of infected cysts in the kidney and liver did not differ. A key finding of this series, given the overall $\mathrm{Cl}$ severity, was the need for invasive intervention in the vast majority of the patients, even in those with $<5-\mathrm{cm}$ infected cysts.

Since the design of a control group to best address the roles of TKV and TLV in Cl development would be very difficult, requiring patients with similar additional risk factors for $\mathrm{Cl}$ but different kidney and/or liver volumes, we approached this issue in the best possible way. We compared TKV and HtTKV in the infected and noninfected kidney groups, comprised of patients with supposedly higher risk for $\mathrm{Cl}$ than the general ADPKD population. This strategy provided a relatively similar background between these two groups, except for the potential differences offered by organ volume. In this context, our data revealed that $\mathrm{KCl}$ was associated with higher TKV and HtTKV, suggesting kidney volume as a risk factor for $\mathrm{KCl}$. Interestingly, history of urinary tract infection has been regarded as an independent prognostic factor for GFR decline in ADPKD patients (22). Notably, the organvolume approach has also been used to estimate risk for nephrolithiasis in ADPKD (23). Our results showed, in addition, that $\mathrm{LCl}$ was also associated with higher TLV, supporting liver volume as a potential risk factor for $\mathrm{LCl}$. Possible explanations for such findings likely include: architectural changes in the renal parenchyma induced by the progressive increase in cystic burden, resulting in areas of outflow obstruction and ectasia that would favor bacterial growth, areas of focal ischemia, changes in the microenvironment driven by local inflammation associated with release of cytokines and chemokines, and increased generation of reactive oxygen species. Taken together, such abnormalities would favor the formation of niches particularly susceptible to infection.

MRI sensitivity was higher than that of $C T$, confirming previous findings (4). PET/CT, in turn, provided the highest sensitivity for $\mathrm{Cl}$ detection. We made adjustments in the PET/CT criteria proposed by Jouret et al. (6) to improve Cl diagnosis. In addition to increased ${ }^{18} \mathrm{FDG}$ activity lining the cyst, detected in $85 \%$ of all episodes, we included a second imaging pattern, characterized by diffuse accumulation within the cyst, which was demonstrated in $60 \%$ of all episodes. In our opinion, performing late image acquisition following furosemide administration, when necessary, improved the contrast between kidney background and the high ${ }^{18}$ FDG uptake by the infected cyst, facilitating diagnosis when the parenchymal signal imposes technical difficulties. The usefulness of this approach in stage 4 and 5 chronic kidney disease, merits further studies. Measurement of another parameter, the SUV $V_{\max }$, has also been shown to contribute to $\mathrm{Cl}$ diagnosis; values $>5.0$ were highly suggestive of infection. In centers where 
PET/CT is not available, we favor an approach using MRI to investigate $\mathrm{Cl}$, particularly when $\mathrm{CT}$ is negative. However, when CT and/or MRI remain inconclusive in a patient without clinical improvement, we recommend rapid referral to a polycystic kidney disease center.

CRP levels are useful in the clinical follow-up of $\mathrm{Cl}$ in ADPKD. In this study, all CRP levels were measured at admission in all patients; however, due to its retrospective nature, the sequential monitoring of CRP was not standardized, limiting the interpretation of the results. On the other hand, it should be mentioned that in the small group in which a second PET/CT was performed, the CRP levels decreased in one patient preceding improvement on PET/CT, as previously reported (7). Further studies should address the role of CRP monitoring in $\mathrm{Cl}$, particularly when evaluating criteria of cure.

Ciprofloxacin was our first-line ATB (24); however, we favored a switch to trimethoprim-sulfamethoxazole in outpatients to avoid quinolone-induced resistance $(25,26)$. We balanced the approach to empirically treat with antibiotics all hospital-admitted ADPKD patients with abdominal pain and fever against the risk of unnecessary treatment in cases of noninfectious complications, such as cyst hemorrhage. Indeed, of 5 patients with cyst hemorrhage, 3 received empirical antibiotics, which were stopped after PET/CT excluded infection. Considering these data, one could consider the restricted use of antibiotics until the release of the initial laboratory and/or imaging results to be a better strategy. This tailored strategy, however, should not be applied to those with sepsis at admission, in whom empirical antibiotics are definitely beneficial. The high severity and mortality associated with $\mathrm{Cl}$ at our institution, should therefore justify empiric antibiotic administration in

\section{References}

1. Bastos AP, Onuchic LF. Molecular and cellular pathogenesis of autosomal dominant polycystic kidney disease. Braz J Med Biol Res 2011; 44: 606-617, doi: 10.1590/S0100879X2011007500068.

2. Pirson $Y$. Extrarenal manifestations of autosomal dominant polycystic kidney disease. Adv Chronic Kidney Dis 2010; 17: 173-180, doi: 10.1053/j.ackd.2010.01.003.

3. Schwab SJ, Bander SJ, Klahr S. Renal infection in autosomal dominant polycystic kidney disease. Am J Med 1987; 82: 714-718, doi: 10.1016/0002-9343(87)90005-2.

4. Sallée M, Rafat C, Zahar JR, Paulmier B, Grunfeld JP, Knebelmann B, et al. Cyst infections in patients with autosomal dominant polycystic kidney disease. Clin J Am Soc Nephrol 2009; 4: 1183-1189, doi: 10.2215/CJN.01870309.

5. Schnelldorfer T, Torres VE, Zakaria S, Rosen CB, Nagorney DM. Polycystic liver disease: a critical appraisal of hepatic resection, cyst fenestration, and liver transplantation. Ann Surg 2009; 250: 112-118, doi: 10.1097/SLA.0b013e3181ad 83dc.

6. Jouret $F$, Lhommel $R$, Beguin $C$, Devuyst $O$, Pirson $Y$, Hassoun Z, et al. Positron-emission computed tomography in patients with clinical signs of potentially severe infection and in those whose initial laboratory and imaging work-up are suggestive of such an etiology.

Despite robust care and follow-up, the mortality rate reached $20.8 \%$. This outcome reflects the severity of $\mathrm{Cl}$ cases referred to our tertiary-care center and suggests some key considerations. First, a surgical approach should be considered early in emphysematous $\mathrm{Cl}(27,28)$; second, cyst drainage should be considered in $\mathrm{Cl}$ events with partial clinical improvement; and third, PET/CT is an outstanding diagnostic tool, but when performed late after ATB initiation, can lead to false-negative results.

Our findings suggest, moreover, that repeating PET/ $\mathrm{CT}$ after $\mathrm{Cl}$ diagnosis may improve management in some situations, such as to confirm cure in patients with $\mathrm{Cl}$ when surgical drainage was indicated, but not feasible/ performed due to technical difficulties. Repeat PET/CI may also benefit ADPKD patients with $\mathrm{Cl}$ in whom documentation of cure is essential for other reasons, such as to initiate chemotherapy or to undergo a surgical procedure. The negative predictive value of PET/CT to exclude $\mathrm{Cl}$, however, needs to be assessed in future studies.

\section{Supplementary Material}

Click here to view [pdf].

\section{Acknowledgments}

We thank Rui T. Barros and Viktoria Woronik for clinical support to the patients included in the present study.

cyst infection diagnosis in patients with autosomal dominant polycystic kidney disease. Clin J Am Soc Nephrol 2011; 6: 1644-1650, doi: 10.2215/CJN.06900810.

7. Jouret $F$, Lhommel $R$, Devuyst $O$, Annet L, Pirson $Y$, Hassoun Z, et al. Diagnosis of cyst infection in patients with autosomal dominant polycystic kidney disease: attributes and limitations of the current modalities. Nephrol Dial Transplant 2012; 27: 3746-3751, doi: 10.1093/ndt/gfs352.

8. Pei Y, Obaji J, Dupuis A, Paterson AD, Magistroni R, Dicks E, et al. Unified criteria for ultrasonographic diagnosis of ADPKD. J Am Soc Nephrol 2009; 20: 205-212, doi: 10.1681/ASN. 2008050507.

9. Qian Q, Li A, King BF, Kamath PS, Lager DJ, Huston J III, et al. Clinical profile of autosomal dominant polycystic liver disease. Hepatology 2003; 37: 164-171, doi: 10.1053/jhep. 2003.50006.

10. Katano K, Kakuchi Y, Nakashima A, Takahashi S, Kawano M. Efficacy of diffusion-weighted magnetic resonance imaging in detecting infected cysts in a case of polycystic kidney disease. Clin Nephrol 2011; 75 (Suppl 1): 24-26.

11. Rosset A, Spadola L, Ratib O. OsiriX: an open-source 
software for navigating in multidimensional DICOM images. J Digit Imaging 2004; 17: 205-216, doi: 10.1007/s10278004-1014-6.

12. Chapman AB, Bost JE, Torres VE, Guay-Woodford L, Bae KT, Landsittel $D$, et al. Kidney volume and functional outcomes in autosomal dominant polycystic kidney disease. Clin J Am Soc Nephrol 2012; 7: 479-486, doi: 10.2215/CJN.09500911.

13. Kanaan N, Goffin E, Pirson Y, Devuyst O, Hassoun Z. Carbohydrate antigen 19-9 as a diagnostic marker for hepatic cyst infection in autosomal dominant polycystic kidney disease. Am J Kidney Dis 2010; 55: 916-922, doi: 10.1053/ j.ajkd.2009.12.023.

14. Sklar AH, Caruana RJ, Lammers JE, Strauser GD. Renal infections in autosomal dominant polycystic kidney disease. Am J Kidney Dis 1987; 10: 81-88.

15. Gibson P, Watson ML. Cyst infection in polycystic kidney disease: a clinical challenge. Nephrol Dial Transplant 1998; 13: 2455-2457, doi: 10.1093/ndt/13.10.2455.

16. Migali G, Annet L, Lonneux M, Devuyst O. Renal cyst infection in autosomal dominant polycystic kidney disease. Nephrol Dial Transplant 2008; 23: 404-405, doi: 10.1093/ndt/gfm665.

17. Telenti A, Torres VE, Gross JB Jr, Van Scoy RE, Brown ML, Hattery RR. Hepatic cyst infection in autosomal dominant polycystic kidney disease. Mayo Clin Proc 1990; 65: 933942, doi: 10.1016/S0025-6196(12)65154-4.

18. Christophe JL, van Ypersele de Strihou C, Pirson Y. Complications of autosomal dominant polycystic kidney disease in 50 haemodialysed patients. A case-control study. The U.C.L. Collaborative Group. Nephrol Dial Transplant 1996; 11: 1271-1276, doi: 10.1093/ndt/11.7.1271.

19. Gilbert BR, Cerqueira MD, Eary JF, Simmons MC, Nabi HA, Nelp WB. Indium-111 white blood cell scan for infectious complications of polycystic renal disease. J Nucl Med 1985; 26: $1283-1286$.

20. Kaim AH, Burger C, Ganter CC, Goerres GW, Kamel E, Weishaupt D, et al. PET-CT-guided percutaneous puncture of an infected cyst in autosomal dominant polycystic kidney disease: case report. Radiology 2001; 221: 818-821, doi: 10.1148/radiol.2213010445.

21. Hsu CT, Chang HR, Lee JK, Weng JH, Kao PF. FDG PET/ CT repeatedly demonstrated hepatic cyst infection in a patient with autosomal dominant polycystic kidney disease. Clin Nucl Med 2013; 38: e188-e190, doi: 10.1097/ RLU.0b013e318266d056.

22. Rule AD, Torres VE, Chapman AB, Grantham JJ, GuayWoodford LM, Bae KT, et al. Comparison of methods for determining renal function decline in early autosomal dominant polycystic kidney disease: the consortium of radiologic imaging studies of polycystic kidney disease cohort. J Am Soc Nephrol 2006; 17: 854-862, doi: 10.1681/ASN.2005070697.

23. Nishiura JL, Neves RF, Eloi SR, Cintra SM, Ajzen SA Heilberg IP. Evaluation of nephrolithiasis in autosomal dominant polycystic kidney disease patients. Clin J Am Soc Nephrol 2009; 4: 838-844, doi: 10.2215/CJN.031006 08.

24. Elzinga LW, Golper TA, Rashad AL, Carr ME, Bennett WM Ciprofloxacin activity in cyst fluid from polycystic kidneys. Antimicrob Agents Chemother 1988; 32: 844-847, doi: 10.1128/AAC.32.6.844.

25. Schwab SJ, Weaver ME. Penetration of trimethoprim and sulfamethoxazole into cysts in a patient with autosomaldominant polycystic kidney disease. Am J Kidney Dis 1986; 7: 434-438.

26. Elzinga LW, Golper TA, Rashad AL, Carr ME, Bennett WM Trimethoprim-sulfamethoxazole in cyst fluid from autosomal dominant polycystic kidneys. Kidney Int 1987; 32: 884-888, doi: 10.1038/ki.1987.290

27. Sooraj YS, Nainan GK, Joseph F, Thara P. Emphysematous polycystic renal infection. Indian J Nephrol 2010; 20: 205206, doi: 10.4103/0971-4065.73457.

28. Yamanouchi M, Ubara Y, Takaichi K. Emphysematous cyst infection in autosomal dominant polycystic kidney disease. Clin Exp Nephrol 2012; 16: 505-506, doi: 10.1007/s10157012-0622-6. 